\title{
Light and Electron Microscopic Study of the Effect of Orlistat on the Liver of Adult Male Albino Rats and the Possible Protective Role of $\beta$-Carotene
}

\author{
Sahar Youssef \\ Anatomy Department, Faculty of Medicine for Girls, Al-Azhar University, Cairo, Egypt \\ Email: sahar_sayed@yahoo.com
}

How to cite this paper: Youssef, S. (2018) Light and Electron Microscopic Study of the Effect of Orlistat on the Liver of Adult Male Albino Rats and the Possible Protective Role of $\beta$-Carotene. Forensic Medicine and Anatomy Research, 6, 20-36. https://doi.org/10.4236/fmar.2018.62003

Received: March 5, 2018

Accepted: April 21, 2018

Published: April 24, 2018

Copyright $\odot 2018$ by author and Scientific Research Publishing Inc. This work is licensed under the Creative Commons Attribution International License (CC BY 4.0).

http://creativecommons.org/licenses/by/4.0/ (c) (i) Open Access

\begin{abstract}
Orlistat is a safe and effective drug to treat obesity by acting as a pancreatic and gastric lipase inhibitor, resulting in reduction in fat absorption. There is also concern that it may be linked with an increased threat of serious hepatic incidents. The present work was carried out to study the effect of orlistat on the histological, immunohistochemical and electron microscopic structure of the liver in the adult male albino rats and the possible protective role of $\beta$-carotene administration. Forty adult albino rats were subjected to experiment for two weeks as follows: group 1 (control), group II, each animal received $0.52 \mathrm{mg} / \mathrm{kg}$ bw/day $\beta$-Carotene, group III, each animal received orlistat $32 \mathrm{mg} / \mathrm{kg} /$ day, and group IV received $\beta$-Carotene, 1hour before the administration of orlistat at same dose of group II \& III. The liver from each animal was dissected out and processed for histological, (light and electron microscopic study). The result of Hep-Par1 for immunohistochemistry was statistically analyzed. The results showed that orlistat treated group displayed variable disturbance of liver architecture, from dilatation, congested central and portal veins, branching of bile ductules, mononuclear cellular infiltration, areas of hemorrhages, cytoplasmic vacuolation and pyknotic nuclei. The most obvious changes were that degenerative changes in hepatocytes led to depletion of glycogen content of hepatocytes. Hep Par-1 revealed a wide area of negative immune expression around the central vein and in some hepatocytes. Other hepatocytes expressed weak reaction. Ultrastructure examination displayed hepatocytes with swollen mitochondria and others with an electron-dense matrix. The combined treatment of $\beta$-Carotene and orlistat led to a marked improvement in most of the previously mentioned changes. It was concluded that orlistat-induced hepatic toxicity. Thus, clinicians should cautiously monitor
\end{abstract}


their patients for signs of hepatic dysfunction. Using an antioxidant such as $\beta$-Carotene decreased the toxicity of orlistat.

\section{Keywords}

Liver, Orlistat, Ultrastructure, $\beta$-Carotene

\section{Introduction}

Obesity has been involved in many chronic conditions, comprising cardiovascular disease, diabetes mellitus, hypertension, obstructive sleep apnoea, hyperlipidaemia, fatty liver disease and malignancy [1]. Orlistat was official by the Food and Drug Administration (FDA) in 1998 for weight loss. Crucially, orlistat is the first gastrointestinal lipase inhibitor for the management of obesity [2]. Orlistat is a dominant inhibitor of gastrointestinal lipase. Following oral administration, orlistat is excreted nearly completely in the feces within three to five days, undergoes slight systemic absorption and displayed no accumulation [3] [4]. Orlistat deactivated gastrointestinal lipase, decreasing the absorption of dietary fat [5]. Significantly, orlistat diminished fat absorption by binding covalently to the serine residue of the active site of gastric and pancreatic lipases. It was suggested that orlistat administration with fat comprising foods partly prevented the hydrolysis of triglycerides, hence decreasing the consequent absorption of monoglycerides and free fatty acids [6]. The XENDOS study, which compared orlistat to placebo in over 3000 patients, showed statistically significant and sustained weight loss at the end of a 4-year study [7]. Orlistat, $120 \mathrm{mg}$ in combination with diet has been shown to reduce liver fat in patients with type 2 diabetes [8] and reduce inflammation and fibrosis in non-alcoholic steatohepatitis [9]. Significantly, orlistat improved blood pressure, insulin resistance and serum lipid levels [10]. Moreover, orlistat has also been shown to decrease total cholesterol and low-density lipoprotein levels independent of weight loss [11]. Recent investigations recommended that orlistat was more effective in dropping weight and lipid profile than metformin [12]. However, the most commonly gastrointestinal adverse effects of orlistat include diarrhea, flatulence, bloating, abdominal pain and dyspepsia [10]. Orlistat is metabolized in the gastrointestinal tract and its direct damaging effect is publicized in intestinal villi of animal models [13]. Pancreatitis associated with the use of orlistat was also recorded by several investigators [14] [15]. The effect of orlistat on the liver is still a matter of controversy. Moreover, the mechanism of orlistat-induced hepatotoxicity remains indistinct.

The daily multivitamins with orlistat are co-prescribed to inhibit fat-soluble vitamin deficits [16]. At present, the hepatoprotective drugs are insufficient, even though the recently developed drugs have been used often have adverse effects. Therefore, a special interest has been directed to the use antioxidants in the treatment and may have an important role in maintaining health [17]. 
$\beta$-Carotene is considered a carotenoid which performed as a vitamin A precursor, and it is the most abundant form of vitamin A in fruits and vegetables

[17]. It has antioxidant capability by scavenging free radicals and quenching singlet oxygen [18]. Animals are unable to synthesize carotenoids de novo, and they are dependent on the diet as a source of these compounds [19]. Van Poppel suggested that diet fruits and vegetables rich in $\beta$-Carotene or high blood levels of $\beta$-Carotene are linked with a reduced risk of cancer at lung and stomach [20]. Previous investigation demonstrated that $\beta$-carotene has not been revealed to precipitate vitamin A toxicity [21].

The Hepatocyte Paraffin antigen-1 (HepPar-1) is a mitochondrial urea cycle antigen. Several reports have been increasingly used Hep-Par1 as a positive biomarker for hepatic distinction [22].

Taken together, the incidence of obesity has been markedly increased worldwide, and many people are interested to decrease their weight by the administration of orlistat. The current work aimed to study the histological, immunohistochemical and electron microscopic changes that may occur in the liver of adult male albino rats after treatment with orlistat and to evaluate the possibility of protection of $\beta$-carotene.

\section{Materials and Methods}

\subsection{Animals}

This study was carried out on forty adult male albino rats. They were weighed between 150 - $200 \mathrm{~g}$ each. Rats were housed in separate well-ventilated cages, under standard conditions, with free access to standard diet and water ad libitum. Rats were kept at a controlled temperature of $25^{\circ} \mathrm{C} \pm 1^{\circ} \mathrm{C}$ and under a $12 \mathrm{~h}$. light: $12 \mathrm{~h}$. dark schedule. Rats were left for two week before the start of experiments for acclimatization. Rats used in this study bought from the Central Animal House, Faculty of Medicine, Assiut University. All animal procedures were in agreement with the principles guidelines for the carefulness and use of experimental animals by the Committee for the Purpose of Supervision of Experiments on Animals. Furthermore, all animals processes were in accordance to the National Institute of Health $(\mathrm{NIH})$ protocol and they agreed by the Institutional Ethics Committee of Assiut University.

\subsection{Drugs and Chemicals}

Orlistat was purchased as a capsule with a trade name (REGIMAX) Produced by Global Napi Pharmaceuticals (GNP), Penta Pharma, Egypt.

\subsection{Experimental Design}

The animals were divided into four equal groups (10 rats each). Rats were subjected to experiment for two weeks as follows:

1) Group I (Control group): Rats received $1 \mathrm{ml}$ of fish oil, the vehicle for orlistat, orally by a gastric tube once daily. 
2) Group II ( $\beta$-carotene group): Rats received $\beta$-carotene supplementation at $0.52 \mathrm{mg} / \mathrm{kg}$ body weight (BW)/day [23].

3) Group III (Orlistat treated group): Rats received orlistat dissolved in fish oil orally by a gastric tube at a dose of $32 \mathrm{mg} / \mathrm{kg} / \mathrm{day}$ [24]. The orlistat dose was calculated according to the interspecies dose conversion scheme by Paget and Branes [25].

4) Group IV (Orlistat \& $\beta$-carotene group): Rats received $\beta$-carotene supplementation at $0.52 \mathrm{mg} / \mathrm{kg}$ bw/day, one hour before the administration of orlistat dose of $32 \mathrm{mg} / \mathrm{kg} /$ day. Both orlistat and $\beta$-carotene were administered orally.

\subsection{Histological Study}

The liver specimens was dissected out and divided into two halves by longitudinal section. One half was processed for light microscopic investigation, whereas the other was processed for electron microscopic investigation. The liver specimens were fixed in a $10 \%$ formalin solution and they were processed and embedded in paraffin wax by routine protocol. 3 - $4 \mu \mathrm{m}$ thick sections were obtained and stained with Haematoxylin and Eosin to study the general structure [26]. Periodic acid Schiff's reaction (PAS) technique was used for the study of mucopolysaccharides and polysaccharides [27].

\subsection{Immunohistochemical Study}

For the immunohistochemical study, $4 \mu \mathrm{m}$ paraffin sections were mounted on charged slides coated on polylysine. The immunohistochemical stain were performed for localization of hepatocyte mitochondrial membrane antigen using hepatocyte paraffin-1 (Hep Par-1). The liver sections were deparaffinized in xylene, rehydrated in descending grades of alcohol. The antigen retrieval was carried out by treating the liver sections with $0.1 \mathrm{~mol} / \mathrm{L}$, citrate buffer at $\mathrm{pH} 6.0$ for ten minutes in a microwave at $100^{\circ} \mathrm{C}$ for twenty minutes, and subsequently left to cool at room temperature for another twenty minutes. To block the endogenous peroxidase, the slides were incubated in hydrogen peroxide, $3 \%$ for five minutes. Then, they were washed twice in PBS for five minutes each. Sections were incubated with the primary antibody, anti-HepPar-1 mouse monoclonal antibody (Lab vision, USA). The primary antibody was diluted at 1:100 overnight at room temperature [28]. After rinsing in PBS, the liver tissues were incubated with a biotin bound to secondary antibody (DAKO-EnVision Plus System-HRP). The peroxidase reaction was visualized using diaminobenzidine (DAB). The sections were counterstained with Mayer's hematoxylin before examination under microscope followed by dehydration, clearing and then mounting. Normal liver tissues were used as the positive controls, however, the negative control liver slides were achieved by omitting the primary antibody and subsequently, no immunostaining was occurred. Finally, the Hep Par-1 positivity appeared as coarsely granular brown cytoplasmic staining. The investigation and photography were conducted at the Mycology and Biotechnology Unit, 
Al-Azhar University, Cairo, Egypt.

\subsection{Morphometric Study}

Quantitative morphometric measurements were done by using the image analyser Leica Qwin standard, 500c, England. The image analyser was calibrated automatically to transform the measurement units, the pixels formed by the image analyser program into real micrometer units. Using the measuring field menu, the area $\%$ and standard measuring frame were chosen from the parameters. The area percentage for HepPar-1 immunoreaction in hepatocytes was measured using the objective lens of magnification 40 , total magnification, $\times 400$. Ten readings were achieved in each specimen and the mean values were done.

\subsection{Statistical Analysis}

The data obtained were subjected to statistical analysis using SPSS statistical software (SPSS for Windows, version 13.0). Comparison between groups was made using ANOVA. All data were expressed as mean $( \pm)$ SD. The Results were considered significant when the $\mathrm{P}$ value less than $0.05(\mathrm{P}<0.05)$.

\subsection{Electron Microscopy Study}

Liver Specimens for electron microscopy were immediately fixed in $2.5 \%$ gluteraldehyde for 24 hours. Then liver specimens were washed in 3 - 4 changes of cacodylate buffer ( $\mathrm{pH}$ 7.2) for 20 minutes each. The postfixation was done in cold $1 \%$ osmium tetroxide for 2 hours and they were washed in four changes of cacodylate buffer for twenty minutes each. The specimens were dehydrated by using ascending grades of alcohol and clearing were carried out in propylene oxide. Embedding was carried out in Epon 812 using gelatin capsules. For polymerization, the embedded samples were kept in an incubator at $35^{\circ} \mathrm{C}$ for one day, incubator at $45^{\circ} \mathrm{C}$ for another day, and finally incubator at $60^{\circ} \mathrm{C}$ for three days. The semi-thin sections of $1-\mu \mathrm{m}$ thickness were cut and they stained with toluidine blue. Ultrathin sections $(500$ - 800) from selected areas of trimmed blocks were collected on copper grids. The ultrathin sections were then contrasted in uranyl acetate for ten minutes and in lead acetate for five minutes. Sections were examined by a transmission electron microscope and JEOL 100 CX, (Japan) and photographed at $80 \mathrm{kV}$ at the Assiut University, Electron Microscopy Unit.

\section{Results}

\subsection{Histological Results}

The liver sections stained with H\&E of the control group (group I) showed the normal histological architecture. The cells appeared as cords radiating from central veins. The hepatic cells appeared polygonal in shape with rounded vesicular nuclei and a granular acidophilic cytoplasm. The cords were separated from each other by blood sinusoids. The hepatic sinusoids appeared as narrow spaces 
between the hepatic plates which lined by flat endothelial cells (Figure 1(A)).

The liver sections stained with $\mathrm{H} \& \mathrm{E}$ of the $\beta$-Carotene-treated group (group II) showed a normal hepatic structure. The hepatocytes were arranged in the form of plates radiating from the central vein (Figure 1(B)). The hepatocytes were polyhedral with acidophilic granular cytoplasm. They had large rounded central vesicular nuclei (Figure 1(B)).
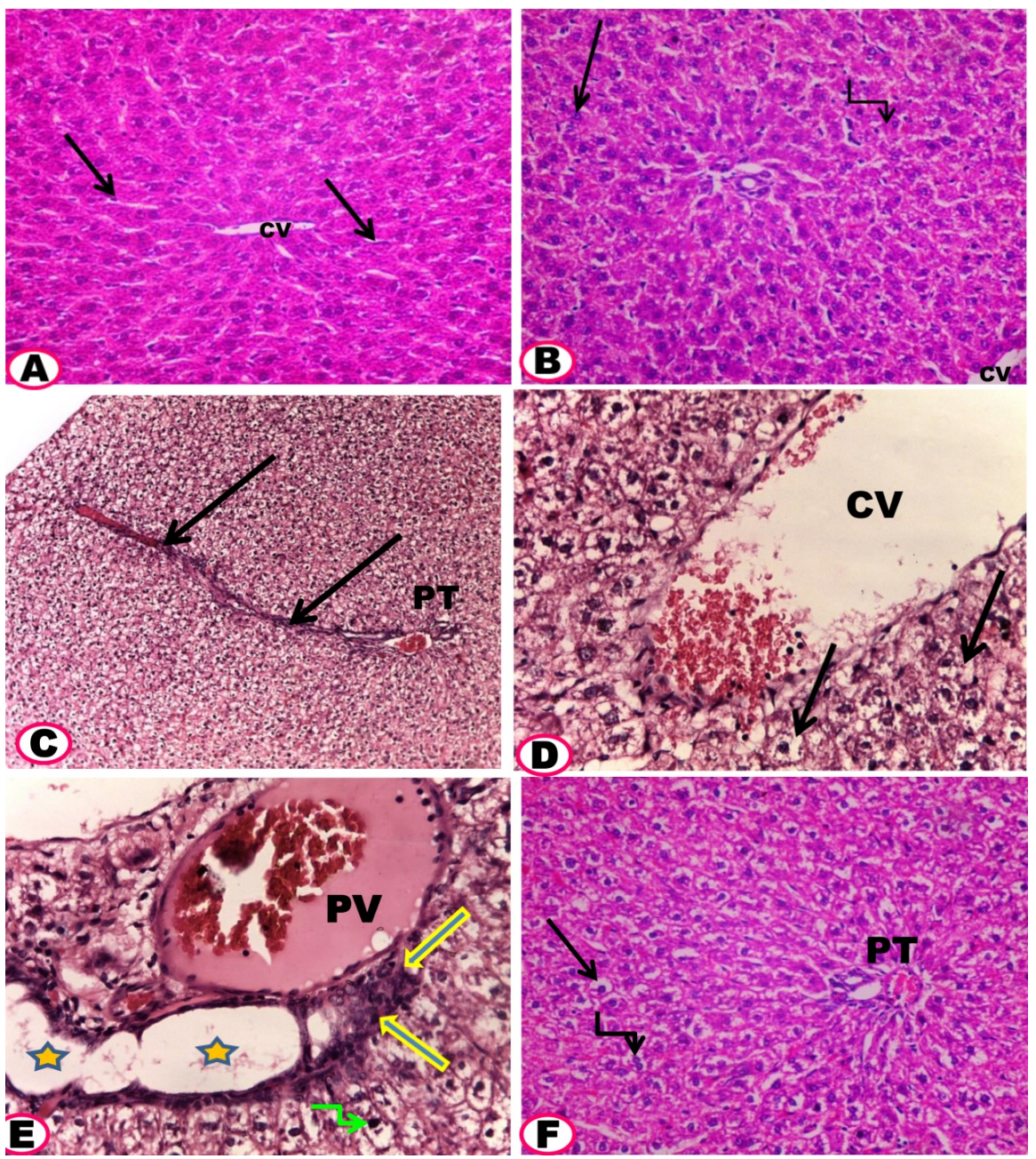

Figure 1. Photomicrographs of liver sections. (A) Control liver section showed radially arranged hepatocyte forming cords around the central vein $(\mathrm{CV})$ and separated by blood sinusoid (arrows). H\&E, $\times 400$. (B) $\beta$-carotene liver section showed cords of polyhedral hepatocytes radiating from central vein $(\mathrm{CV})$. Note, the hepatocytes with acidophilic granular cytoplasm (curved arrow) and central rounded vesicular nuclei (straight arrow). $\mathrm{H} \& \mathrm{E}, \times 400$. (C) Orlistat liver section showed a large area of cellular infiltrations (arrows) extend to portal tract $(\mathrm{PT})$. H\&E, $\times 100$. (D) Orlistat liver section showed dilated and congested central vein (CV). The cytoplasm of hepatocytes is vacuolated (arrows). H\&E, $\times 400$. (E) Orlistat liver section showed dilated congested portal vein (PV) and cellular infiltration (straight arrows) and proliferation of bile duct (stars). Some of hepatocytes showed pyknotic nuclei (curved arrow). H\&E, $\times 400$. (F) Orlistat and $\beta$-carotene liver section showed restoration of liver structure. Note, some hepatocyte with vacuolated cytoplasm (straight arrow). The binucleated hepatocytes (curved arrow) can be seen. H\&E, $\times 400$. 
The liver sections stained with H\&E of the orlistat treated group (group III) showed extensive mononuclear cellular infilteration. The cellular infilteration extend to portal tract (Figure 1(C)). The liver sections showed dilated and congested central veins (Figure 1(D)). The hepatocytes displayed loss of normal hepatic architecture with vacuolated cytoplasm (Figure 1(D)). The dilatation and congested portal veins was also detected. Proliferation of bile ducts, branching of bile ductules, and periportal mononuclear cellular infiltration were also observed. Some hepatocyte nuclei were shrunken and deeply stained (Figure 1(E)).

The liver sections stained with $\mathrm{H} \& \mathrm{E}$ of the orlistat and $\beta$-Carotene -treated group displayed an apparent improvement in the hepatocytes compared with those treated with orlistat. The hepatocytes appeared normal but some cells still revealed vacuolated cytoplasm. The blood sinusoids were still dilated (Figure 1(F)). Binucleated hepatocytes were detected (Figure 1(F)).

\subsection{Histochemical Results}

The control liver section stained with PAS revealed a substantial amount of polysaccharides granules in the ground cytoplasm of hepatocytes (Figure 2(A)).

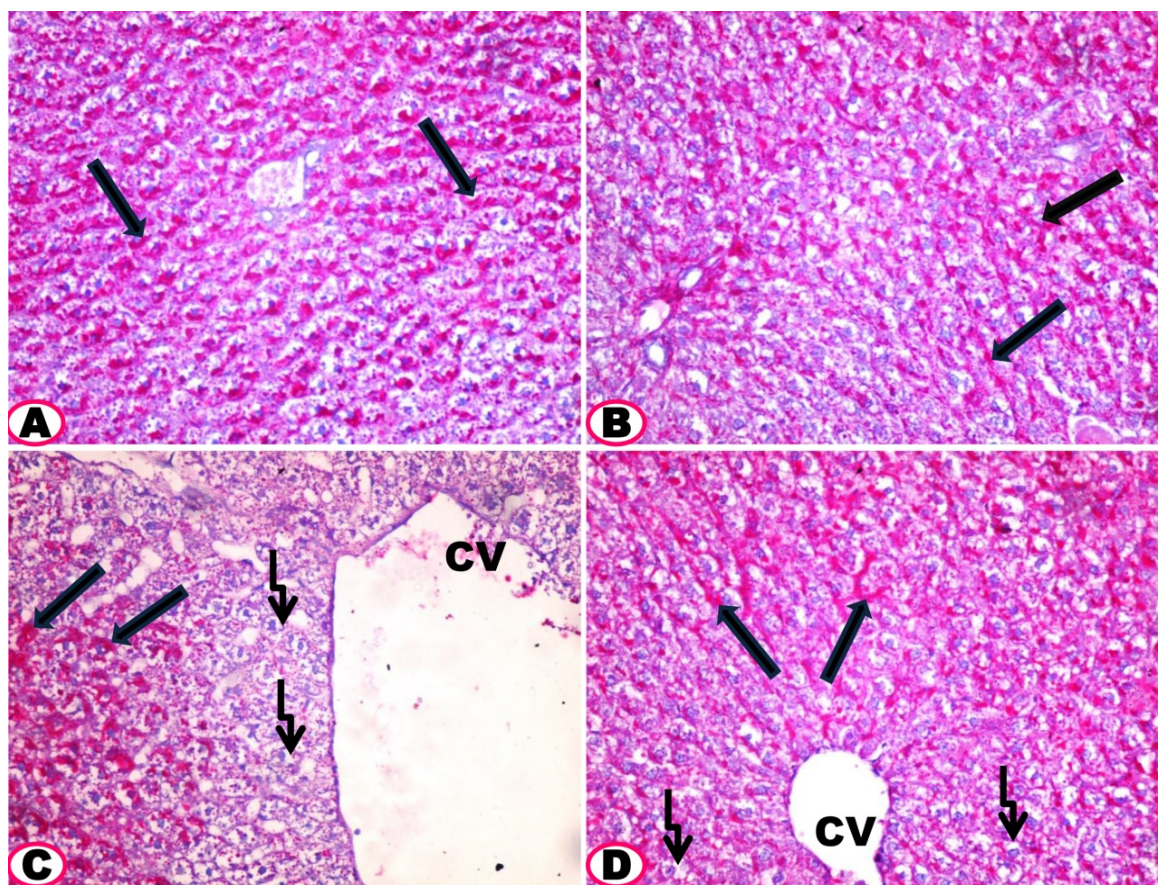

Figure 2. Photomicrographs of liver sections of different studied groups. (A) Control Liver section showed normal extensive amount of glycogen deposition in the ground cytoplasm of hepatocytes (arrows). (B) $\beta$-carotene liver section showed normal glycogen deposition in hepatocytes (arrows). (C) Orlistat liver section showed Large area with negative PAS reaction (curved arrows). Other hepatocytes with substantial amount of PAS positive reaction (straight arrows). (D) Orlistat and $\beta$-carotene liver section showed heterogeneous intensity of PAS reaction. Some hepatocytes revealed a strong PAS positive reaction (straight arrows). Others displayed a moderate PAS reaction (curved arrow). (A)-(D) PAS reaction, $\times 400$. 
The $\beta$-Carotene liver section stained with PAS showed normal glycogen distribution in the cytoplasm of hepatocytes (Figure 2(B)).

The orlistat liver section stained with PAS revealed areas with negative PAS reaction (Figure 2(C)). Reduction of polysaccharide content in many hepatocytes was observed, however, few hepatocytes still exhibited extensive amount of polysaccharides (Figure 2(C)).

The orlistat and $\beta$-Carotene liver section stained with PAS displayed heterogeneous intensity of PAS reaction. Some hepatocytes revealed a strong PAS reaction (Figure 2(D)). While other hepatocytes exhibited a moderate PAS reaction (Figure 2(D)).

\subsection{Immunohistochemical Results}

Immunohistochemical results for Hep Par-1 in the control liver section showed strong positive Hep Par-1 immunoreaction in the form of coarsely scattered granules throughout the hepatocytes cytoplasm with narrow negative immunoreaction around the central vein (Figure $3(\mathrm{~A})$ ).

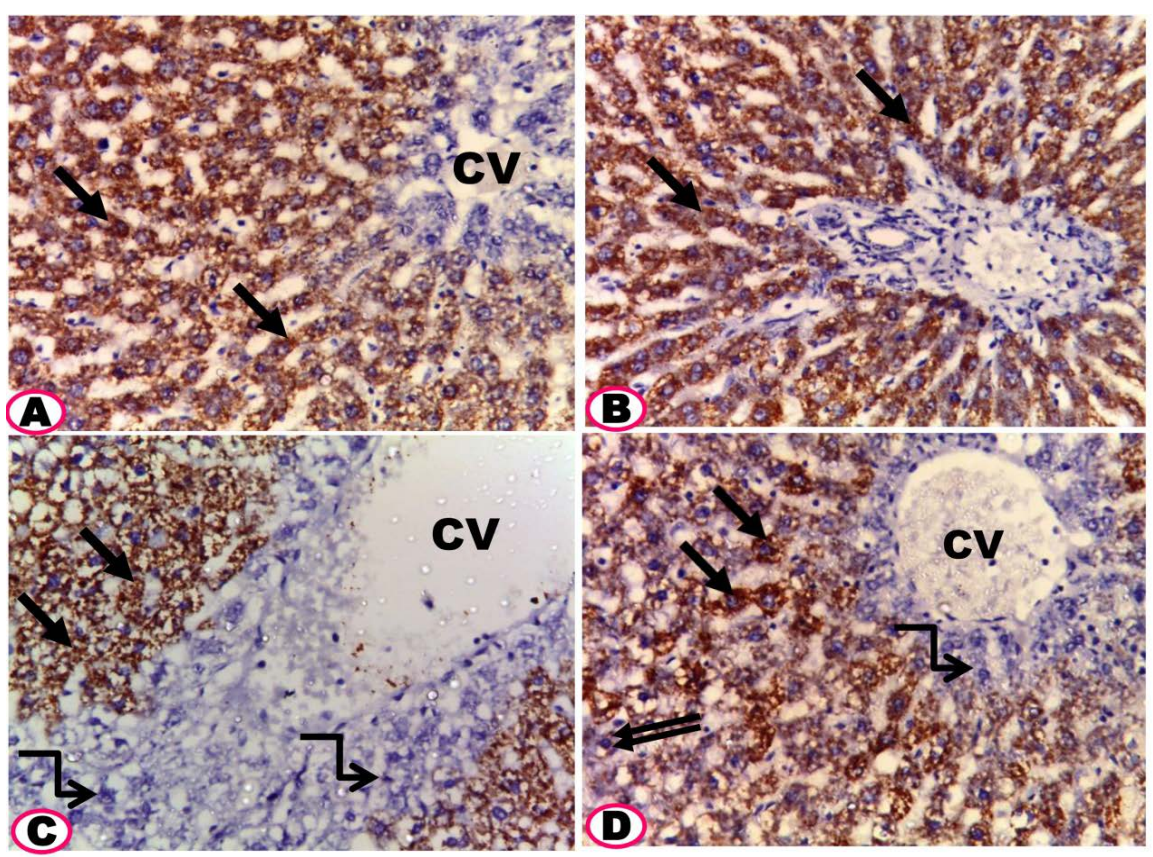

Figure 3. Photomicrographs for the immunohistochemical detection of hepatocyte marker (Hep-par1) in liver sections in different studied groups. (A) Control liver section showed strong brown granular cytoplasmic expression of Hepatocytes (arrows) with narrow negative immunoreaction zone around central vein (CV). (B) $\beta$-carotene liver section showed strong granular immunoreactivity in the cytoplasm of hepatocytes (arrows). (C) Orlistat liver section showed a wide area of negative immune expression around the central vein (CV) and in some hepatocytes (curved arrows). Other hepatocytes express weak reaction (straight arrows). (D) Orlistat and $\beta$-carotene liver section showed variable heterogenous immunoreactivity. Some hepatocytes revealed strong granular cytoplasmic expression (straight arrows), other express weak reaction (double arrow). Note, moderate area of negative immune reaction (curved arrow) around the central vein (cv) can be seen. (A)-(D) Hep-Parlimmunohistochemical, $\times 400$. 
The $\beta$-Carotene liver section for Hep Par-1 displayed strong expression of Hep Par-1 in the form of granular cytoplasmic immunoreaction of hepatocytes with negative immunoreaction around in the portal tract (Figure 3(B)).

The orlistat liver section for Hep Par-1 revealed a wide area of negative immune expression around the central vein and in some hepatocyte was observed (Figure 3(C)). Other hepatocytes expressed weak reaction (Figure 3(C)).

The Orlistat and $\beta$-Carotene liver section for Hep-Par1 exhibited patchy positive immunoreactivity for Hep Par-1. Some hepatocytes revealed strong granular cytoplasmic expression (Figure 3(D)). Other hepatocytes expressed weak immune reaction for Hep Par1. In addition, moderate area of negative immune reaction around the central vein can be seen (Figure 3(D)).

\subsection{Electron Microscopy Results}

The ultrastructure of the control group showed normal hepatic cell with rounded or oval euchromatic nucleus and prominent nucleolus. The well-developed nuclear membrane was detected. The cytoplasm was crowded with cell organelles, the most numerous, the mitochondria and the rough endoplasmic reticulum. The organelles were situated around the nucleus (Figure 4(A)).

The ultrastructure of $\beta$-Carotene group showed hepatocytes with euochromatic rounded nuclei. The cytoplasm contained many mitochondria and rough endoplasmic reticulum (Figure 4(B)).

The ultrastructure of orlistat group showed some of the hepatocytes appeared with small darkly stained nuclei (Figure 4(C), Figure 4(D) and Figure 5(C)). Crucially, the mitochondria were numerous, large or giant that they are clearly observed (Figure 5(A), Figure 5(B)). Obviously, some of them had electron-dense matrix as well as dense granules (Figure 5(A), Figure 5(B)). The cytoplasm showed dilated proliferated endoplasmic reticulum (Figure 4(C)), some vacuoles (Figure 4(C), Figure 4(D) and Figure 5(C)) and lipid droplets were also detected (Figure $4(\mathrm{C})$ ).

The ultrastructure of Orlistat and $\beta$-Carotene group revealed most of the hepatocytes appeared more or less normal with rounded nucleus (Figure 5(D)). The numerous mitochondria and slightly dilated rough endoplasmic reticulum were detected (Figure 5(D)).

\subsection{Mean Area \% of Hep-Par 1 Immunoreactivity}

Morphometric analysis of $\beta$-Carotene group (Group II) showed that the mean area percentage of Hep-Par 1 revealed almost normal value which was statistically insignificant compared with group I $(\mathrm{P}>0.05)$. Expressively, marked decrease in the mean area percentage of Hep-Par lin the group III of orlistat treated group (Table 1 and Figure 6) which was statistically significant compared with the mean value of the control group (Group I). Crucially, manifest increase in group IV which was statistically significant as compared with the orlistat group (Table 1 and Figure 6). 


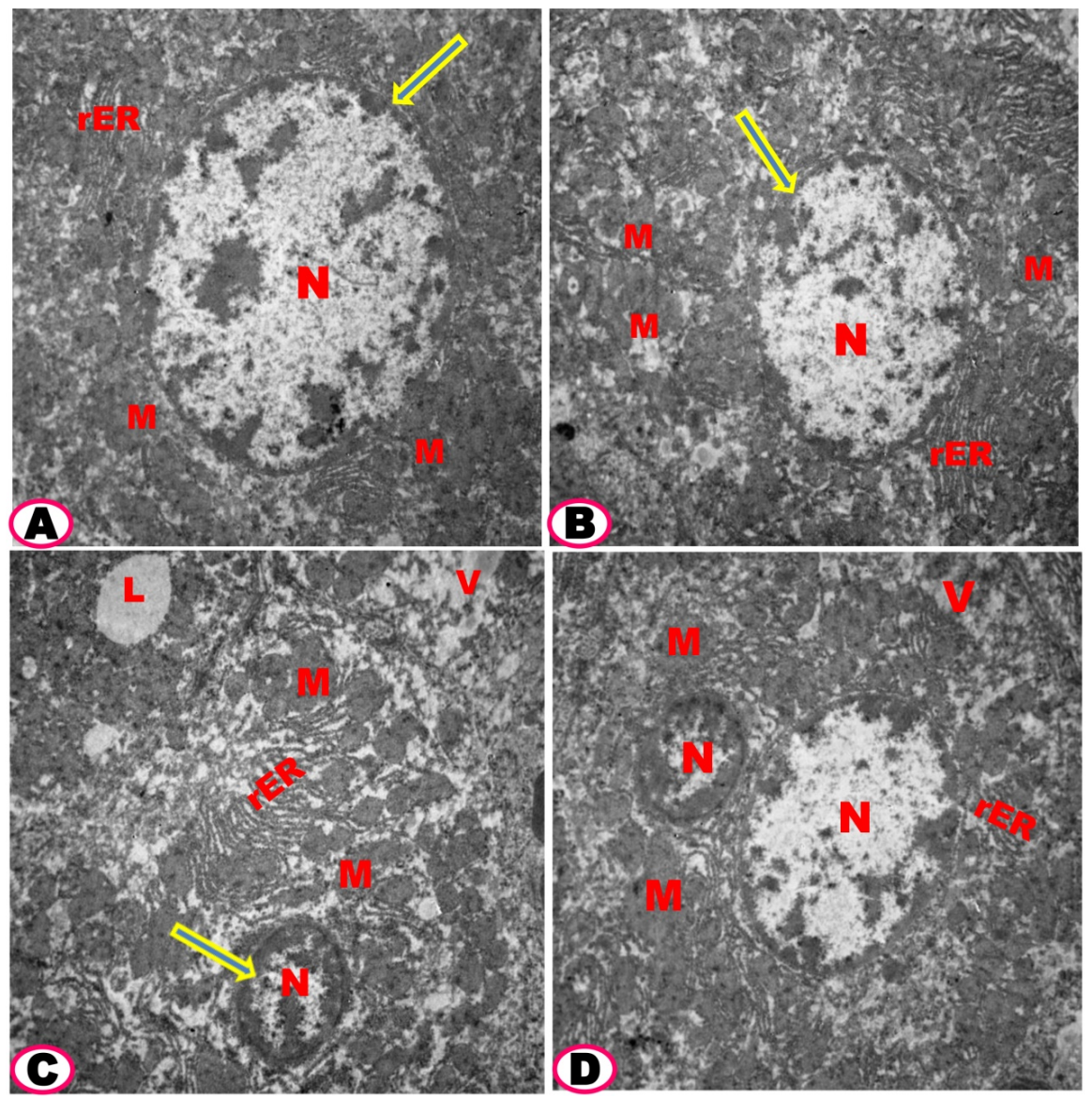

Figure 4. An electron photomicrographs of ultrathin sections in liver of rat. (A) Control group showed normal hepatocytes with large rounded euchromatic nucleus $(\mathrm{N})$. Note, the well-developed nuclear membrane (arrow). The cytoplasm contains profuse amount of rough endoplasmic reticulum $(\mathrm{rER})$ and many mitochondria $(\mathrm{M}) . \mathrm{EM} \times 5800$. (B) $\beta$-carotene group showed the hepatocyte with oval nucleus $(\mathrm{N})$ and regular nuclear membrane (arrow). The cytoplasm contains many mitochondria $(\mathrm{M})$ and rough endoplasmic reticulum $(\mathrm{rER}) . \mathrm{EM} \times 5800$. (C) Orlistat group showed hepatocyte with small electron dense nucleus $(\mathrm{N})$. Note, the nucleus with chromatin condensation (arrow). The cytoplasm contains numerous swollen mitochondria $(\mathrm{M})$, lipid droplet (L), dilated rough endoplasmic reticulum $(\mathrm{rER})$, and few vacuolations $(\mathrm{V})$. EM $\times 5800$. (D) Orlistat group showed binuclear hepatocytes. Note, they were not identical in size, one of them appeared small with peripheral heterochromatic condensation. Note, variable size of mitochondria $(\mathrm{M})$ and few of vacuolations $(\mathrm{V})$ can be seen. $\mathrm{EM} \times 5800$.

\section{Discussion}

In the present study, the administration of orlistat induced alterations in the light and electron microscopic structures of the rat liver. The light microscopic structure orlistat group displayed congested portal and central veins. Crucially, the divergent blood vessel dilatation could recommend metabolic disturbances in the liver, thus signifying a potential harm effect of orlistat on the liver. Significantly, an increase in mononuclear cellular infiltration was also detected in the periportal areas. This result has been described by some researchers [29]. The inflammatory cellular infiltration observed in the orlistat treated group were in 


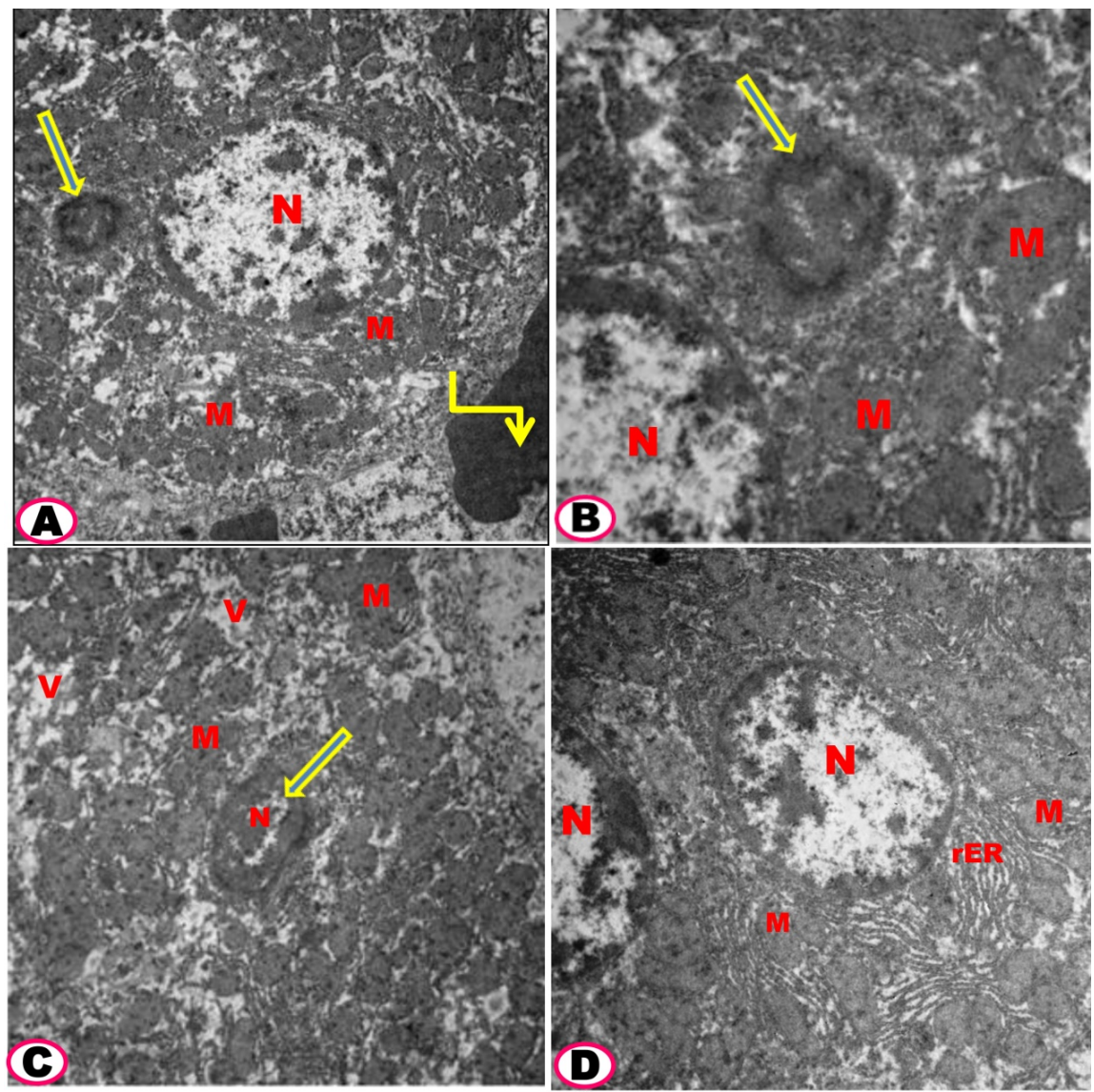

Figure 5. An electron photomicrographs of ultrathin sections in liver of rat. (A) Orlistat group showed rounded nucleus $(\mathrm{N})$, giant mitochondria $(\mathrm{M})$. Note, the swollen mitochondria with electron dense matrix (straight arrow) and blood (curved arrow) are observed. $\mathrm{EM} \times 5800$. (B) Orlistat group showed the higher magnification of Figure $5(\mathrm{~A})$ to show part of nucleus $(\mathrm{N})$ and the swollen mitochondria $(\mathrm{M})$ and increased electron dense matrix (arrow). EM $\times 14,000$. (C) Orlistat group showed hepatocyte with small electron dense nucleus $(\mathrm{N})$. Note, the nucleus (arrow) with chromatin condensation. The cytoplasm contains numerous swollen mitochondria (M) and few vacuolations (V). EM $\times 5800$. (D) Orlistat and $\beta$-carotene showed two adjacent hepatocytes. Notice the presence of numerous Mitochondria $(M)$ and slightly dilated rough endoplasmic reticulum (rER). $\mathrm{EM} \times 7200$.

agreement with investigators who proposed that reactive oxygen species with lipid peroxidation products may cause mitochondrial dysfunction, which can lead to apoptosis and necrosis, activating a cascade leading to fibrosis and collagen deposition [30]. Previous investigators explained that the inflammatory mediators made in hepatocytes, such as TNF- $\alpha$, are most expected to act in a paracrine manner to favor liver injury and hence mononuclear cellular infiltration [31].

In the current study, orlistat group showed liver damage in the form of hepatocellular necrosis as manifested by enlargement of the hepatocytes with cytoplasmic vacuolations and pyknotic nuclei. The present results are in agreement with previous reports that suggested that orlistat treatment were associated with limited cases of severe hepatic adversative effects such as cholelithiasis, cholostatic 
Table 1. Comparison of the mean area percentage of Hep-Par1 between experimental groups.

\begin{tabular}{|c|c|c|c|c|}
\hline \multirow[b]{2}{*}{ Groups } & \multicolumn{4}{|c|}{$M e a n \pm S D(\%)$} \\
\hline & Control group & $\beta$-Carotene & Orlistat group & $\begin{array}{c}\beta \text {-Carotene \& } \\
\text { Orlistat }\end{array}$ \\
\hline Area \% & $77.65 \pm 3.66$ & $77.47 \pm 3.75$ & $58.85 \pm 7.95^{\star}$ & $70.75 \pm 5.23^{\star *}$ \\
\hline
\end{tabular}

The values are expressed as mean \pm SD. Non-significant between group I \& group II. ${ }^{*}$ Test of significance between control rats and orlistat treated rats at $\mathrm{p}<0.001$. ${ }^{* *}$ Test of significance between orlistat treated rats and $\beta$-Carotene \& Orlistat treated rats at $\mathrm{p}<0.001$.

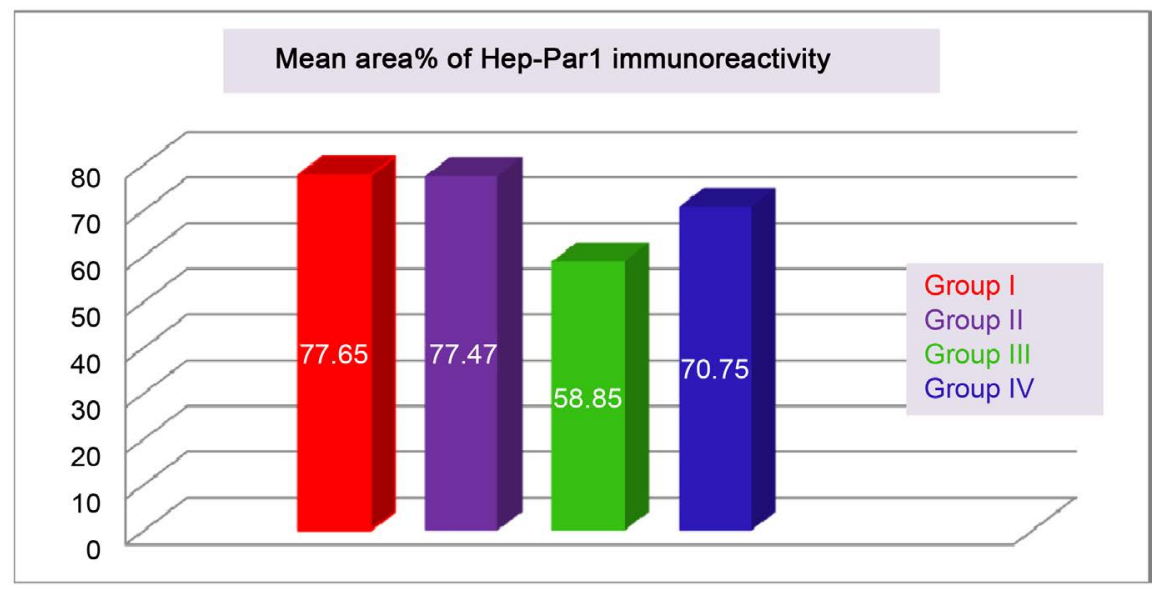

Figure 6. Showing the mean value of area percentage of Hep-Par1 immunoreactivity among studied groups.

hepatitis and subacute liver failures [14]. Importantly, FDA received thirty two cases reports of serious liver injury in patients using orlistat, including 6 cases of liver failure from 1999 to 2008 [32]. In contrast, Orlistat $120 \mathrm{mg}$ in combination with diet has been shown to reduce liver fat in patients with type 2 diabetes [8] and non-alcoholic steatohepatitis, and reduce inflammation and fibrosis in non-alcoholic steatohepatitis [9]. Clinically, orlistat was linked with pancreatitis in some cases. Orlistat was connected with acute pancreatitis with no sign of biliary disease in several cases [14]. Crucially, the most obvious associations between orlistat and pancreatitis appeared to be due to cholelithiasis and increased alcohol consumption by obese participant.

In the present study, bile ductules proliferation observed in the orlistat treated rats. Some researchers recommended that the ductular reactions that happen in response to various liver damages [33].

In the present research, improvement of orlistat was well demonstrated in rats treated with $\beta$-carotene. The liver tissues had preserved in the orlistat and $\beta$-carotene group. The hepatocytes were relatively normal, with vesicular nuclei. The portal areas were not apparently infiltrated with inflammatory cells. In agreement with present assumption, a previous study reported that two thirds of a supplemental dose of $\beta$-carotene will be absorbed during orlistat treatment; this may be sufficient to achieve physiologic levels of $\beta$-carotene. It has been 
shown to prevent the oxidant-mediated activation of inflammatory signaling. $\beta$-carotene removed superoxide radicals and it played an important role in defending cells from these radicals [34].

In the current work, PAS-stained sections of the control group showed normal content of glycogen granules evidenced by a strong PAS reaction. In contrast, the orlistat treated group showed focal weak PAS positive reaction indicating depletion of glycogen. Sakr and his colleague suggested that the reduction in carbohydrate contents could be due to increased stress on the liver, leading to the consumption of high energy in an attempt to diminish or balance the pressure exerted on it [35]. Other researchers suggested that damages carbohydrate metabolism might be due to the inhibition of the intestinal absorption of nutrients and inhibition of hepatic gluconeogenesis [36].

In the present study, all the studied groups of Hep-Par1 sections showed a negative immunoreactive zone around the central veins which were narrow in the control and and $\beta$-carotene group. In contrast, very wide negative immunoreactive area in the orlistat group. Previous investigators recommended that the comparative cellular hypoxia that disturbed mitochondrial metabolic pathways in the level of hepatocytes nearby to the central vein might clarify the negative immunoreactive of this area [37].

In this study, the electron microscopic investigations of orlistat group displayed obvious ultrastructure changes confirmed the light microscopic results. Some cells showed the nuclei often appeared with irregular shapes and chromatin condensation. Other damaged cells seemed with pyknotic nuclei and vacuolated cytoplasm. Moreover, the most obvious degenerative changes appeared in the form of dilated rough endoplasmic reticulum. Definitely, the endoplasmic reticulum was especially liable to the free radical attack, because it is considered as a site of radical production. In addition, its membrane is rich in polyunsaturated fatty acids which are vulnerable to free radical attack. Therefore, the degenerative changes in the endoplasmic reticulum might be due to increased oxidative stress [38].

In the present work, the ultrastructure study of orlistat treated rat showed the electron density of the mitochondrial matrix and swollen mitochondria. The ultrastructure of the mitochondria and its function were definitely impaired. In accordance with present study, other researchers documented that the electron density of the mitochondrial matrix has been attributed to oxidative stress. Then the active substance initially in the mitochondria correlated to apoptosis, including cytochrome $\mathrm{c}$ was released into the cytoplasm [39]. The swollen mitochondria are the major morphological changes in the mitochondrial injury [40]. In addition, Johar \& coworker suggested that apoptosis could be followed by mitochondrial swelling, endoplasmic reticulum dilatation and lysosomal rupture before reduction and termination of nuclei [41]. Other study, in diabetic rat stated that, swelling mitochondria by about $28 \%$ as compensation of reduced mitochondrial ATP production and membranes injured by hydroxyl radicals [42]. 
The present work proved that co-administration of $\beta$-carotene was effective in decreasing the harmful effect of orlistat in the liver of male adult albino rats. This was detected by both light and electron microscopic studies. This perfection might be secondary to the antioxidant ability of $\beta$-carotene, which attacks reactive oxygen species (ROS) and thus neutralizes their detrimental effects on the tissues. Fukuzawa and coworker reported that $\beta$-Carotene acts as an antioxidant by scavenging free radicals and quenching singlet oxygen [18]. It was found that $\beta$-Carotene could have a radical scavenging effect prevented ethanol-induced liver damage and it increased the reduced glutathione (GSH) concentration in rats [43]. Recent report suggested that $\beta$-carotene had distinguished ameliorative effect against arsenic prompted toxicity in albino mice intervened by its antioxidant and antigenotoxic properties [44]. The effective role of $\beta$-carotene supplementation was studied previously and it was reported that the ethanol-induced hepatic depletion of vitamin A can be corrected by $\beta$-carotene supplementation per day [45].

\section{Conclusion}

Clinicians should be aware and carefully monitor their patients on orlistat medication for signs of hepatic dysfunction. The administration of $\beta$-carotene might protect against liver damage produced by orlistat.

\section{References}

[1] Kang, J.G. and Park, C.Y. (2012) Anti-Obesity Drugs: A Review about Their Effects and Safety. Diabetes \& Metabolism Journal, 36, 13-25. https://doi.org/10.4093/dmj.2012.36.1.13

[2] Heal, D.J., Gosden, J. and Smith, S.L. (2012) What Is the Prognosis for New Centrally-Acting Anti-Obesity Drugs? Neuropharmacology, 63,132-146.

https://doi.org/10.1016/j.neuropharm.2012.01.017

[3] Zhi, J., Melia, A. T., Funk, C., Viger-Chougnet, A., Hopfgartner, G. and Lausecker, B. (1996) Metabolic Profiles of Minimally Absorbed Orlistat in Obese/Overweight Volunteers. The Journal of Clinical Pharmacology, 36, 1006-1011. https://doi.org/10.1177/009127009603601104

[4] Sjostrom, L., Rissanen, A., Andersen, T., Boldrin, M., Golay, A. and Koppeschaar, H.P. (1998) Randomised Placebo-Controlled Trial of Orlistat for Weight Loss and Prevention of Weight Regain in Obese Patients. European Multicentre Orlistat Study Group. The Lancet, 352, 167-172. https://doi.org/10.1016/S0140-6736(97)11509-4

[5] Hogan, S., Fleury, A., Hadvary, P., Lengsfeld, H., Meier, M.K. and Triscari, J. (1987) Studies on the Antiobesity Activity of Tetrahydrolipstatin, A Potent and Selective Inhibitor of Pancreatic Lipase. International Journal of Obesity (London), 11, 35-42.

[6] Guerciolini, R. (1997) Mode of Action of Orlistat. International Journal of Obesity and Related Metabolic Disorders, 21, S12-S23.

[7] Torgerson, J.S., Hauptman, J., Boldrin, M.N. and Sjostrom, L. (2004) XENical in the Prevention of Diabetes in Obese Subjects (XENDOS) Study: A randomized Study of Orlistat as an Adjunct to Lifestyle Changes for the Prevention of Type 2 Diabetes in Obese Patients. Diabetes Care, 27, 155-161. https://doi.org/10.2337/diacare.27.1.155 
[8] Kelley, D.E., Kuller, L.H., McKolanis, T.M., Harper, P., Mancino, J. and Kalhan, S. (2004) Effects of Moderate Weight Loss and Orlistat on Insulin Resistance, Regional Adiposity, and Fatty Acids in Type 2 Diabetes. Diabetes Care, 27, 33-40. https://doi.org/10.2337/diacare.27.1.33

[9] Hussein, O., Grosovski, M., Schlesinger, S., Szvalb, S. and Assy, N. (2007) Orlistat Reverses Fatty Infiltration and Improves Hepatic Fibrosis in Obese Patients with Nonalcoholic Steatohepatitis (NASH). Digestive Diseases and Sciences, 52, 2512-2519. https://doi.org/10.1007/s10620-006-9631-1

[10] Siebenhofer, A., Horvath, K., Jeitler, K., Berghold, A., Stich, A.K. and Matyas, E. (2009) Long-Term Effects of Weight-Reducing Drugs in Hypertensive Patients. Cochrane Database of Systematic Reviews, 3, CD007654.

[11] Muls, E., Kolanowski, J., Scheen, A. and L Van Gaal ObelHyx Study Group (2001) The Effects of Orlistat on Weight and on Serum Lipids in Obese Patients with Hypercholesterolemia: A Randomized, Double Blind, Placebo-Controlled Multicentre Study. International Journal of Obesity, 25, 1713-1721. https://doi.org/10.1038/sj.ijo.0801814

[12] Song, J., Ruan, X., Gu, M., Wang, L., Wang, H. and Mueck, A.O. (2017) Effect of Orlistat or Metformin in Overweight and Obese Polycystic Ovary Syndrome Patients with Insulin Resistance. Gynecological Endocrinology, 24, 1-5.

[13] Caner, M., Dogruman, H., Taskin, E., Kandil, A. and Demirci, C. (2005) Effects of Orlistat and Its Relationship with Nitric Oxide in the Small Intestinal Mucosa. Chinese Journal of Physiology, 48, 217-222.

[14] Filippatos, T.D., Derdemezis, C.S, Gazi, I.F., Nakou, E.S., Mikhailidis, D.P. and Elisaf, M.S. (2008) Orlistat-Associated Adverse Effects and Drug Interactions: A Critical Review. Drug Safety, 31, 53-65. https://doi.org/10.2165/00002018-200831010-00005

[15] Kose, M., Emet, S., Akpinara, T.S. and Ilhanc, M. (2015) An Unexpected Result of Obesity Treatment: Orlistat-Related Acute Pancreatitis. Case Reports in Gastroenterology, 9, 152-155. https://doi.org/10.1159/000430433

[16] Padwal, R.S. and Majumdar, S.R. (2007) Drug Treatments for Obesity: Orlistat, Sibutramine, and Rimonabant. The Lancet, 369, 71-77. https://doi.org/10.1016/S0140-6736(07)60033-6

[17] Saini, R.K., Nile, S.H. and Park, S.W. (2015) Carotenoids from Fruits and Vegetables: Chemistry, Analysis, Occurrence, Bioavailability and Biological Activities. Food Research International, 76, 735-750. https://doi.org/10.1016/j.foodres.2015.07.047

[18] Fukuzawa, K., Inokami, Y. and Tokumura, A. (1998) Rate Constants for Quenching Singlet Oxygen and Activities for Inhibiting Lipid Peroxidation of Carotenoids and Alpha-Tocopherol in Liposomes. Lipids, 33, 751-756.

https://doi.org/10.1007/s11745-998-0266-y

[19] Fraser, P.D. and Bramley, P.M. (2004) The Biosynthesis and Nutritional Uses of Carotenoids. Progress in Lipid Research, 43, 228-265. https://doi.org/10.1016/j.plipres.2003.10.002

[20] Van Poppel, G. (1993) Carotenoids and Cancer: An Update with Emphasis on Human Intervention Studies. European Journal of Cancer, 29A, 1335-1344. https://doi.org/10.1016/0959-8049(93)90087-V

[21] Handelman, G.J., van Kuijk, F.J.G.M., Chatterjee, A. and Krinsky, N.I. (1991) Characterization of Products Formed during the Autoxidation of $\beta$-Carotene. Free Radical Biology \& Medicine, 10, 427-437. https://doi.org/10.1016/0891-5849(91)90051-4 
[22] Chu, P.G., Ishizawa, S., Wu, E. and Weiss, L.M. (2002) Hepatocyte Antigen as a Marker of Hepatocellular Carcinoma: An Immunohistochemical Comparison to Carcinoembryonic Antigen, CD10, and Alpha-Fetoprotein. The American Journal of Surgical Pathology, 26, 978-988. https://doi.org/10.1097/00000478-200208000-00002

[23] Peng, H.C., Chen, Y.L., Yang, S.Y., et al. (2013) The Antiapoptotic Effects of Different Doses of $\beta$-Carotene in Chronic Ethanol-Fed Rats. Hepatobiliary Surgery and Nutrition, 2, 132-141.

[24] Cruz Hernandez, C., Oliveira, M., Pescia, G., Moulin, J., Masserey Elmelegy, I., Dionisi, F. and Destaillats, F. (2010) Lipase Inhibitor Orlistat Decreases Incorporation of Eicosapentaenoic and Docosahexaenoic Acids in Rat Tissues. Nutrition Research, 30, 134-140. https://doi.org/10.1016/j.nutres.2009.12.001

[25] Paget, G.E. and Branes, G.M. (1964) Toxicity Tests. In: Laurence, D.R. and Bacharach, A.L., Eds., Evaluation of Drug Activities: Pharmacometrics, Vol. 1, 2nd Edition, Academic Press, London. https://doi.org/10.1016/B978-1-4832-2845-7.50012-8

[26] Bancroft, J.D. and Gamble, M. (2013) Theory and Practice of Histological Techniques. 7th Edition, Churchill Livingstone of Elsevier, Philadelphia, 172-186.

[27] Bancroft, J.D. and Stevens, A. (1996) Theory and Practice of Histotechnological Techniques. 4th Edition, Churchill Livingstone, New York.

[28] Abdelrahman, A.E., Abdel-Aziz, H.R. and Hegazy, A.A. (2015) Immunohistochemical Distinction of Hepatocellular Carcinoma Using Arginase-1, Hepatocyte Paraffin Antigen-1 and Glypican-3. Journal of Tumor, 4, 359-366. https://doi.org/10.17554/j.issn.1819-6187.2016.04.75

[29] AL-Rawi, M.M. (2007) Effect of Trifolium sp. Flowers Extracts on the Status of Liver Histology of STZ-Induced Diabetic Rats. Saudi Journal of Biological Sciences, 14, 21-28.

[30] Atiq, M., Davis, J.C., Lamps, L.W., Beland, S.S. and Rose, J.E. (2009) Amiodarone Induced Liver Cirrhosis. Report of Two Cases. Journal of Gastrointestinal and Liver Diseases, 18, 233-235.

[31] Bu, D.X., Erl, W., De Martin, R., Hansson, G.K. and Yan, Z.-Q. (2005) IKK $\beta$-Dependent NF- $\kappa$ B Pathway Controls Vascular Inflammation and Intimal Hyperplasia. The FASEB Journal, 19, 1293-1295. https://doi.org/10.1096/fj.04-2645fje

[32] U.S. Food and Drug Administration (2010) FDA Drug Safety Communication. Completed Safety Review of Xenical/Alli (orlistat) and Severe Liver Injury. http://www.fda.gov/Drugs/DrugSafety/PostmarketDrugSafetyInformationforPatient sandProviders/ucm213038.htm

[33] LeSage, G., Glaser, S. and Alpini, G. (2001) Regulation of Cholangiocyte Proliferation. Liver, 21, 73-80. https://doi.org/10.1034/j.1600-0676.2001.021002073.x

[34] Jang, S.H., Lim, J.W. and Kim, H.B. (2009) Carotene Inhibits Helicobacter Pylori-Induced Expression of Inducible Nitric Oxide Synthase and Cyclooxygenase-2 in Human Gastric Epithelial AGS cells. Journal of Physiology and Pharmacology, 60, 131-137.

[35] Sakr, S.A., Abdel Samei, H.A. and Soliman, M.E. (2004) Exploring Hepatotoxicity of Benomyl. Histological and Histochemical Study on Albino Rats. Journal of Medical Sciences, 4, 77-83. https://doi.org/10.3923/jms.2004.77.83

[36] Gopumadhavan, S., Mohammed, R., Azeemuddin, M. and Mitra, S.K. (2008) Ameliorative Effect of Partysmart in Rat Model of Alcoholic Liver Disease. Indian Journal of Experimental Biology, 46, 132-137. 
[37] Shehata, A.S., Mohamed, Z.A., Abd El-Haleem, M.R. and Samak, M.A. (2013) Effects of Exposure to Plasticizers Di-(2-Ethylhexyl) Phthalate and Trioctyltrimellitate on the Histological Structure of Adult Male Albino Rats' Liver. Journal of Clinical Toxicology, 3, 4 .

[38] Pradeep, K., Raj Mohan, C.V., Gobianand, K. and Karthikeyan, S. (2010) Protective Effect of Cassia fistula Linn. on Diethylnitrosamine Induced Hepatocellular Damage and Oxidative Stress in Ethanol Pretreated Rats. Biological Research, 43, 113-125. https://doi.org/10.4067/S0716-97602010000100013

[39] Aliev, G., Palacios, H.H., Gasimov, E., Obrenovich, M.E., Morales, L., Leszek, J., et al. (2010) Oxidative Stress Induced Mitochondrial Failure and Vascular Hypoperfusion as a Key Initiator for the Development of Alzheimer Disease. Pharmaceuticals, 3, 158-187. https://doi.org/10.3390/ph3010158

[40] Lu, X., Hamilton, J.A., Shen, J., Pang, T., Jones, D.L. and Potter, R.F. (2006) Role of Tumor Necrosis Factor-Alpha in Myocardial Dysfunction and Apoptosis during Hind Limb Ischemia and Reperfusion. Critical Care Medicine, 34, 484-491. https://doi.org/10.1097/01.CCM.0000199079.64231.C1

[41] Johar, D., Roth, J.C., Bay, G.H., Walker, J.N., Kroczak, T.J. and Los, M. (2004) Inflammatory Response, Reactive Oxygen Species, Programmed (Necrotic-Like and Apoptotic) Cell Death and Cancer. Roczniki Akademii Medycznej W Bialymstoku, 49, 31-39.

[42] Ohkuwa, T., Sato, K. and Naoi, M. (1995) Hydrozyl Radical Formation in Diabetic Rat Induced by Streptozotocin. Life Sciences, 56, 1789-1798. https://doi.org/10.1016/0024-3205(95)00150-5

[43] Lin, W.T., Huang, C.C., Lin, T.J., et al. (2009) Effects of Beta Carotene on Antioxidant Status in Rats with Chronic Alcohol Consumption. Cell Biochemistry and Function, 27, 344-350. https://doi.org/10.1002/cbf.1579

[44] Das, R., Das, A., Roy, A., Kumari, U., Bhattacharya, S. and Haldar, P.K. (2015) $\beta$-Carotene Ameliorates Arsenic-Induced Toxicity in Albino Mice. Biological Trace Element Research, 164, 226-233. https://doi.org/10.1007/s12011-014-0212-4

[45] Ahmed, S., Leo, M.A. and Lieber, C.S. (1994) Interactions between Alcohol and Beta-Carotene in Patients with Alcoholic Liver Disease. The American Journal of Clinical Nutrition, 60, 430-436. https://doi.org/10.1093/ajcn/60.3.430 